\title{
Potencial alelopático do extrato foliar de Annona glabra L. (Annonaceae)
}

\author{
Reginaldo Sadao Matsumoto ${ }^{1,3}$, José Pedro Nepomuceno Ribeiro ${ }^{1}$, Leandro Kenji Takao ${ }^{1}$ e Maria Inês Salgueiro Lima ${ }^{2}$
}

Recebido em 16/02/2009. Aceito em 20/10/2009

RESUMO - (Potencial alelopático do extrato foliar de Annona glabra L. (Annonaceae)). A. glabra cresce em áreas alagadas, formando aglomerados. Este comportamento pode indicar ocorrência de competição com outras espécies ou que existam processos alelopáticos. Neste estudo, os objetivos foram: a) avaliação do potencial alelopático de extratos foliares de A. glabra sobre a germinação e crescimento de outras espécies, e sobre o crescimento de coleóptilos de trigo e b) verificação da presença de grupos de substâncias nos extratos. Os testes qualitativos detectaram triterpenos, taninos e flavonóides. Foi realizada cromatografia de partição líquido:líquido com hexano e acetato de etila. A atividade das frações obtidas foi testada sobre de Lactuca sativa, Echinochloa crus-galli, Euphorbia heterophylla e Ipomoea grandifolia. A fração acetato de etila reduziu a porcentagem de germinação de L. sativa e atrasou o tempo médio de germinação de E. crus-galli além de afetar o crescimento de todas as espécies. Esta fração foi purificada em coluna de sílica, obtendo-se cinco frações (A, B, C, D e E). Para cada uma delas foram preparadas quatro concentrações $(1000 ; 158 ; 79 ; 39,5$ ppm). Os efeitos causados pelas frações foram avaliados com o teste de coleóptilo de trigo. A fração A estimulou o alongamento destes nas três menores concentrações, na maior foi inibitória, B inibiu nas três maiores, $\mathrm{C}$ inibiu apenas na maior e $\mathrm{E}$ não provocou efeito significativo.

Palavras-chave: alelopatia, coleóptilos, plantas infestantes de culturas

\begin{abstract}
Allelopathic potential of leaf extract of Annona glabra L. (Annonaceae)). A. glabra is a wetland species, occurring in dense populations. This behavior may indicate strong competition with other species or the existence of an allelopathic process involved in this distribution. In this study, we aim to: a) evaluate the allelopathic potential of extracts produced by A. glabra leaves on germination and seedling growth of other species and on wheat coleoptile growth and b) verify the presence of substance groups on the extracts and fractions studied. The qualitative test detected triterpenes, tannins and flavonoids. A Liquid:liquid partition chromatography of aqueous extract was done, using hexane and ethyl acetate. The activities of the fractions obtained were tested on germination and initial growth of Lactuca sativa, Echinochloa crus-galli, Euphorbia heterophylla and Ipomoea grandifolia. The ethyl acetate fraction reduced L. sativa germination percentage and E. crus-galli mean germination time and affected the initial growth of all species. This fraction was purified by chromatographic column which resulted in five fractions (A, B, C, D and E). Four concentrations (1000; 158; 79; 39.5 ppm) were prepared for each one. Effects caused by fractions were evaluated by wheat coleoptile tests. Fraction A stimulated elongation at all concentrations except $1000 \mathrm{ppm}$. Fraction B was inhibitory at all concentrations except for the smaller one. C was inhibitory only at the higher concentration and E did not cause a significant effect.
\end{abstract}

Key words: allelopathy, coleoptiles, weed species

\section{Introdução}

Os herbicidas sintéticos têm-se mostrado cada vez menos efetivos na sua ação contra espécies infestantes de culturas, uma vez que o seu uso continuado tem levado à seleção de indivíduos cada vez mais resistentes a esses produtos (Gelmini et al. 2001). Este fenômeno tem levado à busca de novas alternativas, menos agressivas ao meio, como é o caso dos aleloquímicos naturais. Estes têm como vantagens a solubilidade em água, meia vida mais curta e geralmente não são considerados potencialmente tóxicos (Duke 2000).

Os estudos químicos sobre as Annonaceae foram intensificados nos anos 80 e 90, principalmente pela procura de acetogeninas, uma classe de compostos com ampla atividade biológica. E antes disso, as pesquisas centraram suas investigações sobre os alcalóides isoquinolínicos. Foram catalogados cerca de 320 produtos secundários naturais provindos de 150 espécies desta família (Alali et al.1999).

Estudos químicos sobre a Annona glabra L. confirmaram a presença de grupos de substâncias com possível potencial alelopático, como monoterpenóides (Grayson 2000) e diterpenóides, isolados de diferentes partes da planta (Chang et al. 1998; Chen et al. 2000; Yang et al. 2003; Zhang et al.
2004). Chang et al. (2000) e Hsieh et al. (2004) isolaram alcalóides, esteróides e outros diterpenóides. Porém abordagens sobre o seu potencial alelopático ou fitotóxico ainda não haviam sido realizados.

Estudos preliminares não publicados mostraram que extratos aquosos $10 \%(\mathrm{p} / \mathrm{v})$ de folhas de A. glabra exerceram efeitos inibitórios sobre germinação e crescimento inicial de Lactuca sativa L. e no crescimento inicial de três espécies infestantes de culturas: Echinochloa crus-galli (L.) P. Beauv.; Euphorbia heterophylla L. Ipomoea grandifolia (Dammer) O’Donell.

A. glabra é arbustivo-arbórea e cresce nas margens de rios, formando aglomerados. Este comportamento pode indicar que está ocorrendo uma forte competição com outras espécies ou ainda que existam processos alelopáticos envolvidos nesta distribuição. A partir dessas observações e dos testes preliminares realizados anteriormente, foram estabelecidos os seguintes objetivos: A) Avaliar o potencial alelopático de extratos foliares de A. glabra sobre a germinação e crescimento de plântulas de outras espécies, e sobre o crescimento de coleóptilos de trigo. B) Verificar a presença de classes de compostos do metabolismo secundário nos extratos e frações estudados.

\footnotetext{
1 Universidade Federal de São Carlos, Programa de Pós-graduação em Ecologia e Recursos Naturais, Laboratório de Sistemática e Ecologia Química, São Carlos, SP, Brasil

2 Universidade Federal de São Carlos, Departamento de Botânica, Laboratório de Sistemática e Ecologia Química, São Carlos, SP, Brasil

3 Autor para correspondência: sadaomts@gmail.com
} 


\section{Material e métodos}

Material vegetal - Folhas de Annona glabra foram coletadas no estuário

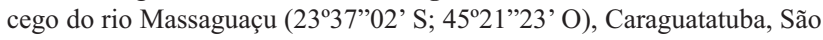
Paulo, Brasil. O material foi seco em estufa a $45^{\circ} \mathrm{C}$ por $48 \mathrm{~h}$. O espécime voucher, número de referência 7503, está depositado no Herbário do Departamento de Botânica da UFSCar (Universidade Federal de São Carlos). Cromatografia de partição líquido:líquido - Um extrato aquoso $10 \%(\mathrm{p} / \mathrm{v})$ foi preparado com $140 \mathrm{~g}$ de folhas secas moídas de A. glabra e água destilada. Após acondicionamento em geladeira por $24 \mathrm{~h}, \mathrm{o}$ extrato foi filtrado à vácuo através de camada dupla de papel filtro $(3 \mu \mathrm{m})$. Em funil de separação, o filtrado foi submetido à cromatografia de partição líquido:líquido (CPLL), na proporção de 1:1, utilizando hexano e posteriormente, acetato de etila. Teste de germinação - Os efeitos dos extratos resultantes da CPLL foram avaliados através da porcentagem e tempo médio de germinação, obtidos mediante as fórmulas propostas por Labouriau \& Valadares (1976). Como espécies alvo foram usadas uma bioindicadora, alface (Lactuca sativa, variedade Grand rapids), e três espécies consideradas plantas infestantes de cultura: capim-arroz (Echinochloa crus-galli); amendoim bravo (Euphorbia heterophylla); corda de viola (Ipomoea grandifolia).

Placas de Petri $(9 \mathrm{~cm})$ foram lavadas e esterilizadas. Cada placa recebeu duas folhas de papel filtro e $5 \mathrm{~mL}$ do extrato. As placas foram acondicionadas em capela até evaporação total dos solventes. Em seguida, os papéis de filtros receberam igual volume de água destilada e 30 sementes foram dispostas em seu interior. Foram vedadas com filme plástico, tampadas e acondicionadas em estufa $\mathrm{DBO}$ a $28^{\circ} \mathrm{C}$, recebendo luz apenas durante a contagem das sementes germinadas.

As sementes germinadas foram contadas e retiradas a cada $12 \mathrm{~h}$, até o final do $10^{\circ}$ dia. Foram consideradas germinadas as sementes cujas radículas apresentavam pelo menos $50 \%$ do tamanho da semente (Ferreira \& Borghetti 2004).

Teste de crescimento inicial - Foram usadas as mesmas espécies alvo e os mesmos procedimentos de lavagem e esterilização dos recipientes do teste de germinação. Caixas plásticas $(12 \times 7 \times 10 \mathrm{~cm})$, contendo duas folhas de papel filtro, foram usadas com uma quantidade de $12 \mathrm{~mL}$ do tratamento. As caixas foram colocadas em capela até a evaporação total dos solventes e reidratadas com o mesmo volume de água destilada. Quinze plântulas (pré-germinadas no escuro, à $28^{\circ} \mathrm{C}$ ) foram acondicionadas em cada caixa. As caixas foram tampadas e colocadas em estufa a $28^{\circ} \mathrm{C}$ com fotoperíodo de $12 \mathrm{~h}$. Após cinco dias, a parte aérea e radícula das plântulas foram medidas.

Em ambos os testes, cada espécie alvo recebeu três tratamentos (controle, contendo água destilada; extrato hexânico; extrato com acetato de etila) no delineamento experimental inteiramente casualizado, com cinco réplicas. Os dados de porcentagem e tempo médio de germinação e comprimento das plântulas foram analisados com ANOVA com pós-teste de Tukey ou Kruskall-Wallis com pós-teste de Dunn. Com intuito de avaliar os efeitos de possíveis resíduos dos solventes após sua secagem, um segundo controle foi executado utilizando somente os solventes como tratamento. Foram utilizados ensaios de germinação e crescimento inicial com as mesmas espécies alvo e a mesma metodologia.

Fracionamento em coluna de sílica gel-Com base nos resultados dos testes de germinação e crescimento inicial da extração líquido:líquido, o extrato com resultados inibitórios (acetato de etila) foi submetido ao fracionamento em coluna de sílica gel (60-200 Mesh). Na composição da fase estacionária da coluna, foi adicionada sílica ao extrato e seco em capela. Como fase móvel foi usado um gradiente de solventes de polaridades crescentes: hexano; hexano/diclorometano (3:1); hexano/diclorometano (1:1); hexano/ diclorometano (1:3); diclorometano; diclorometano/acetato de etila (3:1); diclorometano/acetato de etila (1:1); diclorometano/acetato de etila (1:3); acetato de etila; acetato de etila/ acetona (1:1); acetona; acetona/metanol (1:1); metanol. As frações resultantes foram separadas em frascos de $50 \mathrm{~mL}$.

O conteúdo das frações foi analisado por cromatografia em camada delgada (Cézar et al. 2007), utilizando-se acetato de etila/acetona (1:1) como eluente e vanilina como revelador. As frações iniciais foram reunidas em frações contendo substâncias semelhantes.

Teste em coleóptilo de trigo (Bobylev et al. 2000) - As cinco frações resultantes foram secas, pesadas e resuspendidas com DMSO (dimetilsulfóxido) $1 \% \mathrm{e}$ solução tampão ácido cítrico/hidrogenofostato $\mathrm{pH} 5,6$ contendo $2 \%$ de sacarose. Foram preparadas quatro concentrações: 1 (39,5 ppm);2 (79 ppm); 3 (158 ppm); $4(1000 \mathrm{ppm})$ para cada grupo e sua atividade testada em coleóptilos de trigo.
Sementes de trigo foram germinadas em água destilada a $24^{\circ} \mathrm{C}$, no escuro, por 72 h. Em câmara escura sob luz verde, os coleóptilos das plântulas estioladas de trigo foram cortados, medindo $4 \mathrm{~mm}$. Nos tubos de ensaio foram colocados $2 \mathrm{~mL}$ do devido tratamento e quatro coleóptilos. Os tubos foram lacrados com filme plástico e colocados em centrífuga de rotação $(6 \mathrm{rpm})$, os tubos ficaram posicionados horizontalmente. $\mathrm{O}$ conjunto foi coberto a fim de evitar contato com a luz e mantido em estufa escura a $22^{\circ} \mathrm{C}$. Após $24 \mathrm{~h}$, os coleóptilos foram fotografados e medidos.

Um controle positivo para inibição do crescimento foi feito com cumarina. Este foi preparado usando as seguintes concentrações: 39,$5 ; 79 ; 158$; $500 \mathrm{ppm}$. Não foi usada a concentração de $1000 \mathrm{ppm}$ pela dificuldade de diluição e também porque a concentração 500 ppm já mostrou inibição de $100 \%$ nos testes preliminares. Dois controles negativos foram preparados, um apenas com a solução tampão e um com solução tampão e DMSO.

$\mathrm{O}$ delineamento experimental do teste em coleóptilo de trigo e seus controles foi inteiramente casualizado com seis réplicas. Os dados foram comparados com ANOVA com pós-teste de Tukey. Testes qualitativos para compostos secundários:

Detecção de cumarina - Foi feito um extrato metanólico $10 \%$ (p/v), utilizando pó de folhas de A. glabra e metanol. Utilizou-se como reagente, uma mistura $10 \%(\mathrm{p} / \mathrm{v})$ de hidróxido de sódio e água destilada. Dois $\mathrm{mL}$ do extrato foram colocados em um tubo de ensaio, que foi tampado com papel filtro embebido na solução hidróxido de sódio e levado ao banho-maria por cinco minutos. O papel foi revelado sob luz U.V. $(360 \mathrm{~nm})$ e caso houvesse fluorescência, confirmaria a presença de cumarina (Costa 1982).

Teste para detecção de alcalóides - Foi realizado para verificar-se a presença no extrato metanólico $10 \%$ e nas maiores concentrações das frações de substâncias usadas no teste em coleóptilo. Três reagentes (Dragendorff, Wagner e Mayer) foram utilizados na detecção de alcalóides, cuja presença causa leve turbidez ou precipitados (Dragendorff e Wagner), e a presença de cor marrom (Wagner) e creme (Mayer) (Henriques et al. 2003). Cafeína foi usada como controle positivo (Falkenberg 2003).

Teste para detecção de triterpenos e esteróides - Utilizou-se extrato metanólico $10 \%$ e o reagente de Liebermann-Burchard $(2 \mathrm{~mL}$ de anidrido acético + duas gotas de ácido sulfúrico concentrado). Dois $\mathrm{mL}$ do extrato metanólico foram levados a secura e resuspendidos com $5 \mathrm{~mL}$ de clorofórmio, misturados com carvão ativado e filtrados. O reagente foi adicionado e a coloração verde persistente indicaria a presença de triterpenos e esteróides (Costa 1982).

Detecção de taninos - A presença de taninos foi testada pela reação com solução de cloreto férrico (10\%). A reação procedeu-se no extrato metanólico seco (10\%) e resuspendido em água destilada com o dobro do volume. A cor azul sugere a presença de taninos hidrolisáveis e a verde, de taninos condensados (Costa 1982).

Detecção de flavonóides - A presença destes foi avaliada no extrato metanólico $(10 \%)$ através do reativo de Cianidina, ou Shinoda ( $\mathrm{HCl}$ concentrado e magnésio em pó). Através desta reação, pode-se caracterizar compostos contendo um núcleo alfa-benzopirona, pelo desenvolvimento de cor laranja a vermelha (Falkenberg et al. 2003).

\section{Resultados e discussão}

Após o fracionamento líquido:líquido foram obtidas três frações (aquosa, hexânica e em acetato de etila), tendo sido utilizadas apenas as duas últimas. No teste de germinação com a espécie bioindicadora (L. sativa), comparado ao controle, somente o extrato com acetato de etila reduziu a germinação a $2 \%$. O cálculo do tempo médio de germinação dessa espécie ficou comprometido por apresentar réplicas sem nenhuma germinação, e este valor ( 52,8 horas) foi retirado das análises estatísticas (Tab.1).

Os extratos testados não afetaram a porcentagem de germinação das espécies infestantes de cultura (E.crus-galli, E. heterophylla, I. grandifolia). Somente as sementes de $E$. crus-galli tiveram um atraso no tempo médio de germinação, quando tratadas com extrato de acetato de etila (Tab.1). 
Tabela 1. Valores de porcentagem (\%) e tempo médio de germinação (TM, em horas) das espécies receptoras sob três tratamentos (controle, extrato hexânico e de acetato de etila). As letras diferentes indicam diferenças estatistísticas. L. sativa L e E. heterophylla L foram analisados com ANOVA. E. crus-galli (L.) P. Beauv.e I. grandifolia (Dammer) O’Donell foram analisados com Kruskall-Wallis.

\begin{tabular}{|c|c|c|c|c|c|c|c|}
\hline \multirow{2}{*}{$\begin{array}{l}\text { Espécie receptora } \\
\text { Lactuca sativa L. }\end{array}$} & \multirow{2}{*}{$\frac{\text { Germinação }}{\%}$} & \multicolumn{2}{|c|}{ Controle } & \multicolumn{2}{|c|}{ Extrato hexânico } & \multicolumn{2}{|c|}{ Extrato de acetato } \\
\hline & & 80,66 & A & 70 & A & 2 & - \\
\hline & $\mathrm{TM}$ & 33,54 & A & 35,27 & A & 52,8 & - \\
\hline \multirow[t]{2}{*}{ Echinochloa crus-galli (L.) P. Beauv. } & $\%$ & 75,33 & A & 74,67 & A & 70,83 & A \\
\hline & $\mathrm{TM}$ & 44,03 & A & 46,72 & A & 58,6 & $\mathrm{~B}$ \\
\hline \multirow[t]{2}{*}{ Euphorbia heterophylla L. } & $\%$ & 73,33 & A & 70,66 & A & 71,33 & A \\
\hline & $\mathrm{TM}$ & 28,62 & A & 31,1 & A & 37,01 & A \\
\hline \multirow[t]{2}{*}{ Ipomoea grandifolia (Dammer) O’Donell } & $\%$ & 52,66 & $\mathrm{~A}$ & 52,66 & A & 62,67 & A \\
\hline & $\mathrm{TM}$ & 30,49 & A & 30,49 & A & 30,73 & A \\
\hline
\end{tabular}

O extrato hexânico não afetou significativamente o crescimento das plântulas de alface, porém o extrato acetato de etila inibiu o crescimento da parte aérea quando comparado ao controle, mas não houve diferença significativa para a radícula. O comprimento total, embora menor para o extrato com acetato de etila, não foi significativo em relação ao controle (Tab. 2).

$\mathrm{O}$ extrato acetato de etila inibiu significativamente o crescimento da radícula de E. crus-galli, refletindo na diferença do tamanho total das plântulas entre este tratamento e o controle (Tab.2). As raízes, normalmente, são mais sensíveis aos efeitos alelopáticos do que a germinação e o elongamento do hipocótilo (Chon et al. 2002; Oliveira et al. 2004). O extrato hexânico não afetou as plântulas desta espécie.

O extrato hexânico teve efeito inibitório significativo sobre o crescimento das radículas de E. heterophylla. Este efeito foi ainda mais efetivo com o extrato em acetato de etila, que afetou tanto a radícula quanto a parte aérea, tendo reduzido em $50 \%$ o crescimento total das plântulas em relação ao controle (Tab.2).

$\mathrm{O}$ extrato hexânico não afetou significativamente o crescimento da parte aérea e radícula de I. grandifolia, mas afetou significativamente o tamanho total das plântulas. $\mathrm{O}$ extrato em acetato de etila reduziu significativamente o crescimento da parte aérea e radícula, e por conseqüência, o comprimento total da plântula foi reduzido em $44 \%$ em relação ao controle (Tab.2).

Os solventes não causaram nenhum efeito sobre a germinação e crescimento inicial. Esses resultados indicam que o extrato em acetato de etila tem maior potencial fitotóxico, sendo assim, o mesmo foi submetido a coluna cromatográfica e cromatografia em camada delgada gerando cinco frações de fracionamento (A, B, C, D e E).

As frações geradas (A, B, C, D e E) foram secas e pesadas, e os respectivos valores foram 42,$5 ; 28,9 ; 36$; 64,$1 ; 7,9 \mathrm{mg}$. Com este material foram realizados os testes com coleóptilos de trigo. A fração E devido ao baixo rendimento, não foi preparada na concentração de 1000 ppm (Fig.1). O controle contendo DMSO e so- lução tampão não diferiu do controle contendo somente solução tampão.

Todas as concentrações da fração A deram resultados diferentes do controle, sendo que a concentração 1000 ppm inibiu e as demais estimularam o alongamento dos coleóptilos. Aparentemente, a maior parte, se não todos os compostos orgânicos que são inibitórios em alguma concentração, são estimulantes em menores concentrações (Rice, 1984).

Com exceção da menor concentração (39,5 ppm), todas as concentrações da fração B inibiram a o alongamento dos coleóptilos. Somente a concentração 1000 ppm da fração $\mathrm{C}$ inibiu o elongamento dos coleóptilos comparado ao controle. A maior concentração da fração D (1000 ppm) inibiu o crescimento e as concentrações 79 e 39,5 ppm geraram estímulo no crescimento dos coleóptilos de trigo.

Nenhuma das três concentrações testadas da fração E gerou efeito significativo sobre o alongamento dos coleóptilos. Desta forma as frações A, B, C e D parecem conter substâncias com potencial fitotóxico, sendo mais evidentes nas frações B e C, porém a atividade alelopática só poderá ser melhor avaliada em condições de campo.

As frações B, C e cumarinas comparadas entre si dentro das mesmas concentrações mostraram que na concentração 39,5 ppm, os frações B e C não diferiram entre si, mas foram menos inibitórias que a cumarina no crescimento dos coleóptilos. Na concentração de 79 ppm a fração B obteve resultados intermediários a $\mathrm{C}$ e cumarina, que diferiram entre si. Resultado semelhante a este ocorreu na concentração 158 ppm, e na maior concentração, todas as frações foram semelhantes (Fig.2).

Na comparação entre as concentrações do mesmo grupo, o grupo $\mathrm{C}$ apresentou diferença significativa apenas na maior concentração (1000 ppm). No grupo B, as concentrações 79 e $158 \mathrm{ppm}$ diferiram do controle e de $39,5 \mathrm{ppm}$, que foram iguais entre si. A maior concentração diferiu de todas.

A cumarina diferiu do controle em todas as concentrações, sendo 39,5, 79 e 158 ppm iguais entre si. A concentração 500 ppm (maior concentração) diferiu de todas, não havendo praticamente crescimento algum do coleóptilo (Fig.2). 
Tabela 2. Comprimento ( $\mathrm{mm}$ ) da parte aérea (PA), da radícula (Rad) e total das espécies receptoras sob três tratamentos (controle, extrato hexânico e de acetato de etila). As letras diferentes indicam diferenças estatistísticas. E. heterophylla L. e I. grandifolia (Dammer) O'Donell foram analisados com ANOVA. L. sativa L. e E. crus-galli (L.) P. Beauv. foram analisados com Kruskall-Wallis.

\begin{tabular}{|c|c|c|c|c|c|c|c|}
\hline \multirow{2}{*}{$\frac{\text { Espécie receptora }}{\text { Lactuca sativa L. }}$} & \multirow{2}{*}{$\frac{\text { Parte da planta }}{\text { Rad }}$} & \multicolumn{2}{|c|}{ Controle } & \multicolumn{2}{|c|}{ Extrato hexânico } & \multicolumn{2}{|c|}{ Extrato de acetato } \\
\hline & & 19,03 & $\mathrm{AB}$ & 27,19 & A & 9,49 & $\mathrm{~B}$ \\
\hline & PA & 21,73 & A & 20,79 & A & 14,68 & $\mathrm{~B}$ \\
\hline & Total & 40,76 & $\mathrm{AB}$ & 47,98 & A & 24,17 & $\mathrm{~B}$ \\
\hline \multirow[t]{3}{*}{ Echinochloa crus-galli (L.) P. Beauv. } & Rad & 60,91 & A & 54,39 & $\mathrm{AB}$ & 30,05 & $\mathrm{~B}$ \\
\hline & PA & 28,12 & $\mathrm{~A}$ & 27,39 & A & 27,66 & A \\
\hline & Total & 89,03 & A & 81,78 & $\mathrm{AB}$ & 57,71 & $\mathrm{~B}$ \\
\hline \multirow[t]{3}{*}{ Euphorbia heterophylla L. } & Rad & 61,28 & A & 45,08 & $\mathrm{~B}$ & 19,97 & $\mathrm{C}$ \\
\hline & PA & 60,19 & $\mathrm{~A}$ & 58,94 & A & 43,58 & $\mathrm{~B}$ \\
\hline & Total & 121,48 & A & 104,02 & A & 63,55 & $\mathrm{~B}$ \\
\hline \multirow[t]{3}{*}{ Ipomoea grandifolia (Dammer) O'Donell } & Rad & 37,1 & $\mathrm{~A}$ & 29,35 & A & 12,96 & $\mathrm{~B}$ \\
\hline & PA & 48,8 & A & 43,39 & A & 35,15 & $\mathrm{~B}$ \\
\hline & Total & 85,9 & A & 72,74 & $\mathrm{~B}$ & 48,11 & $\mathrm{C}$ \\
\hline
\end{tabular}

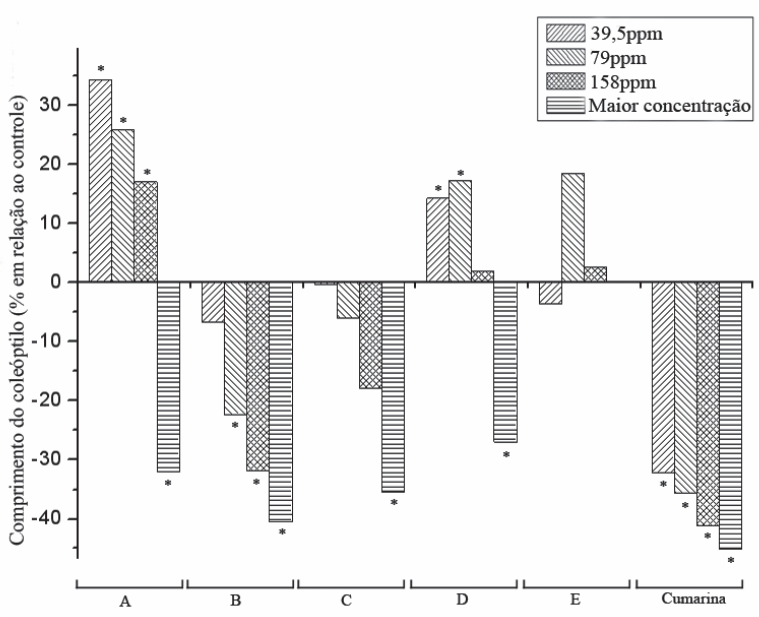

Figura 1. Valores percentuais médios do comprimento dos coleóptilos de trigo em relação ao controle (* - diferença significativa em relação ao controle).

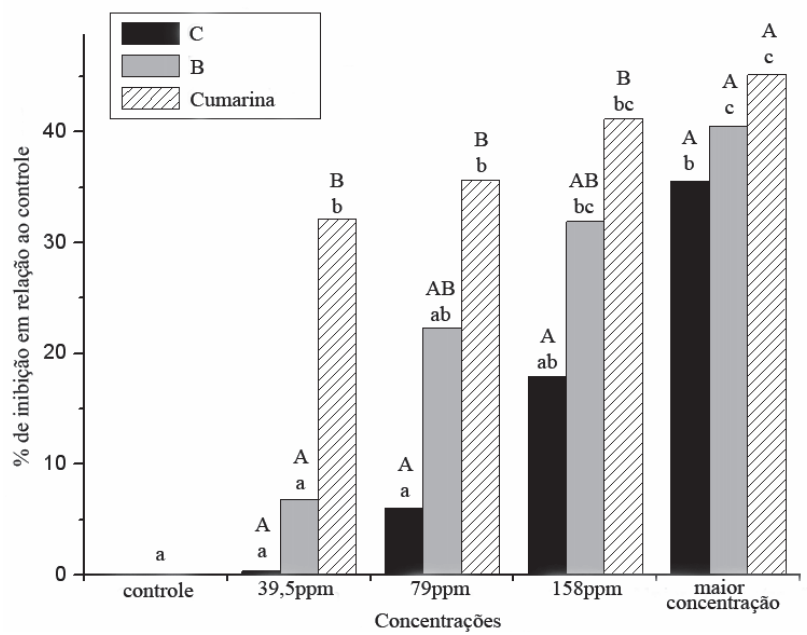

Figura 2. Porcentagem de inibição do comprimento do coleóptilo de trigo nas diferentes concentrações da fração B e C comparadas as de cumarina. As letras maiúsculas são referentes às comparações entre tratamentos na mesma concentração. As letras minúsculas são referentes às comparações entre concentrações da mesma fração. A maior concentração utilizada foi de 1000 ppm para as frações B e C e 500 ppm para a cumarina.
Testes qualitativos para compostos secundários foram negativos para cumarina no extrato metanólico. Resultados positivos foram encontrados para alcalóides, taninos, flavonóides e triterpenos. Os alcalóides também foram detectados na fração D.

Padmaja et al. (1995) e Hsieh et al. (2004) também apontam a existência de alcalóides para $A$. glabra e Henriques $e t$ al. (2003) descrevem várias atividades biológicas para essa classe de substâncias.

Os terpenóides têm sido descritos por suas diversas propriedades, entre elas, a alelopática (Haig 2008). Monoterpenóides e diterpenóides provindos de A. glabra e suas atividades já haviam sido descritos por Chang et al. (1998), Chen et al. (2000), Grayson (2000), Yang et al. (2003) e Zhang et al., 2004. Tripernóides e esteróides também foram detectados no extrato metanólico.

A alelopatia está dentre as atividades biológicas dos taninos (Rawat et al. 1998), e a presença dessa substância foi confirmada no extrato metanólico de A. glabra. Padmaja et al. (1995) citou a presença de quercetina nesta espécie, um flavonóide com atividades variadas como antimicrobiana, antiviral, antitumoral, antioxidante, entre outras. Os flavonóides também podem ter ação alelopática (Zuanazzi \& Montanha 1999). A presença de flavonóides também foi confirmada no extrato metanólico, podendo estar relacionada à quercetina.

Liu et al. (1999) descreveram um fenilpropanóide pertencente a $A$. glabra, que é precursor de substâncias possivelmente alelopáticas, como os compostos fenólicos (Santos 2003).

A confirmação destes compostos no extrato metanólico e a comprovação do potencial alelopático no extrato acetato de etila, principalmente sobre o crescimento inicial das espécies infestantes de culturas, somado aos resultados do fracionamento, no qual, frações com potencial inibitório, inclusive tão ativo quanto a cumarina (grupo B), proporcionam evidências para o possível uso de extratos de $A$. glabra 
como herbicida natural. O isolamento das substâncias nas frações elucidará quais são responsáveis pelo estímulo e pela inibição, e se, uma substância ou a sinergia destas explicam os efeitos encontrados nos resultados, tanto nos extratos acetato, como nos aquosos.

Os testes qualitativos em extrato metanólico mostraram a presença de alcalóides, taninos, flavonóides e triterpenos e a ausência de cumarinas. Os extratos semipurificados de acetato de etila de folhas de Annona glabra comprovaram a existência de substâncias com potencial alelopático, solúveis nesse solvente.

\section{Agradecimentos}

Ao CNPq e a CAPES pela concessão das bolsas. Ao tecnólogo Carlos Aparecido Casali pelo auxílio nos experimentos de laboratório.

\section{Referências bibliográficas}

Alali, F.Q.; Liu, X-X. \& McLaughlin, J.L. 1999. Annonaceous Acetogenins: Recent Progress. Journal of Natural Products 62(3): 504-540.

Bobylev, M.M.; Bobyleva, L. I.; Cutler,.H,G.; Cutler, S.J. \& Strobel, G.A. 2000. Effects of synthetic congeners of the natural product phytotoxins aculosins- 1 and -2 on growth of wheat coleoptiles (Triticum aestivum $\mathrm{L}$. cv. Wakeland). Pp 209-214. In: Anais do X international Symposium on biological control of weeds. Montana State University, Bozeman, Montana, USA. Ed. Neal R. Apencer.

Cézar, I.C.; Braga F.C.; Vianna-Soares; Nunan, E.A.; Barbosa, T. A.F. \& Moreira-Campos, L.M. 2007. Determinação de daidzeina, genisteína e gliciteína em cápsulas de isoflavonas por cromatografia em camada delgada (CCD) e cromatografia líquida de alta eficiência (CLAE). Revista Brasileira de Farmacognosia 17(4): 616-625.

Chang, F-R.; Yang, P-Y.; Lin, J-Y.; Lee, K-H. \& Wu, Y-C. 1998. Bioactive Kaurana diterpenoids from Annona glabra. Journal of Natural Products 61: 437-439.

Chen, C-Y.; Chang, F-R.; Cho, C-P. \& Wu, Y-C. 2000. Ent-Kaurane diterpenoids from Annona glabra. Journal of Natural Products 63(7): 1000-1003.

Chon, S.U.; Choi, S.K.; Jung, S.; Jang, H.G.; Pyo, B.S. \& Kim, S.M. 2002. Effects of alfafa leaf extracts and phenolic allelochemicals on early seedling growth and root morphology of alfafa and barnyard grass. Crop protection 21: 1077-1082.

Costa, A.F. 1982. Farmacognosia III v. 2. ed. Lisboa, Fundação Calouste Gulbenkian.

Duke, S.O.; Dayan, F.E.; Ramafnani, J.G. \& Rimado, A.M. 2000. Natural products as sources of herbicides: current status and future trends. Weed Research 40: 499-505...

Falkenberg, M.B.; Santos, R.I.; Simões, C.M.O. 2003. Introdução à análise fitoquímica. Pp.229-245. In: Simões, C.M.O.; Schenkel,
E.P.; Gosmann, G.; Mello, J.C.P.; Mentz, L.A.; Petrovick, P.R. 2003. Farmacognosia - da planta ao medicamento. Porto Alegre, EFRGS. Ferreira, A.G. \& Borghetti, F. 2004. Germinação do básico ao aplicado. Porto alegre, RS. Artmed Ed.

Gelmini, G.A.; Filho, V.; Novo, R.S. \& Salvo, M.C. 2001. Resistência de biótipos de Euphorbia heterophylla L. aos herbicidas inibidores da enzima ALS utilizados na cultura de soja. Bragantia 60(2): 93-99.

Grayson, D.H. 2000. Monoterpenoids. Natural Product Reports 17: 385-419.

Haig, T. 2008. Allelochemicals in plants. Pp. 63-104. In:. Zeng, R.S.; Mallik A.U \&. Luo A.M (eds). Allelopathy in Sustainable Agriculture and Forestry. London, Springer.

Henriques, A.T; Limberger, R.P.; Kerber, V.A. \& Moreno, P.R. 2003. Acalóides: Generalidades e aspectos básicos. Pp.765-791. In: Simões, C.M.O.; Schenkel, E.P.; Gosmann, G.; de Mello, J.C.P.; Mentz, L.A. $\&$ Petrovick P.R. (eds). Farmacognosia: da planta ao medicamento. Porto Alegre, Ed. da UFRGS.

Hsieh, T-J.; Wu, Y-C.; Chen, S-C.; Huang, C-S. \& Chen, C-Y. 2004. Chemical constituents from Annona glabra. Chinese Chemical Society 51: 869-876.

Labouriau, L.G. \& Valadares, M.B. 1976. On the germination of seeds of Calotropis procera. Anais da Academia Brasileira de Ciências 48: 174-186.

Liu, X.; Pilarinou, E. \& McLaughlin, J.L. 1999. Podaplin: A novel cyclic Prenylated Phenylpropanoid from Annona glabra. Tetrahedron Letters 40: 399-402.

Oliveira, S.C.C.; Ferreira, A.G.; Borguetti. F. 2004. Efeitos alelopáticos de folhas de Solanum lycocarpum A. St.-Hil.(Solanaceae) na germinação e crescimento de Sesamun indicum L. (Pedaliaceae) em solo sob três temperaturas. Acta Botanica Brasilica 18(3): 401-406.

Padmaja, V.; Thankamany, V.; Hara, N.; Fujimoto, Y. \& Hisham, A. 1995. Biological activities of Annona glabra. Etnopharmacology 48: 21-24.

Rawat, M.S.M.; Pant, G.; Prasad, D.; Joshi, R.K. \& Pande, C.B. 1998. Plant growth inhibitors (Proanthocyanidins) from Prunus armeniaca. Biochemical Systematic and Ecology 26: 13-23.

Rice, E. L. 1984. Allelopathy. New York, Academic Press.

Santos, R.I.P.R. 2003. Metabolismo básico e origem dos metabólitos secundários. Pp.403-434. In: Simões, C.M.O.; Schenkel, E.P.; Gosmann, G.; de Mello, J.C.P.; Mentz, L.A. \& Petrovick P.R. (eds). Farmacognosia: da planta ao medicamento. Porto Alegre, Ed. da UFRGS.

Yang, N.Y; Tian, L.J.; Meng, Z.M. \& Han, Y. 2003. A new Diterpenoid Dimern from Annona glabra. Chinese Chemical Letters 14(1): 58-61.

Zhang, Y-H.; Peng, H-Y; Xia, G-H.; Wang, M-Y. \& Han, Y. 2004. Anticancer effect of two diterpenoid compounds isolated from Annona glabra Linn. Acta Pharmacologica Sinica 25(7): 937-942.

Zuanazzi, J.A.S. \& Montanha, J.A. 1999. Flavonóides. Pp.577-614. In: Simões, C.M.O.; Schenkel, E.P.; Gosmann, G.; de Mello, J.C.P.;. Mentz, L.A. \& Petrovick P.R. (eds). Farmacognosia: da planta ao medicamento. Porto Alegre, Ed. da UFRGS. 